\title{
Venus transit 2004: Illustrating the capability of exoplanet transmission spectroscopy
}

\author{
P. Hedelt ${ }^{1,2}$, R. Alonso ${ }^{3}$, T. Brown ${ }^{4}$, M. Collados Vera ${ }^{5}$, H. Rauer ${ }^{6,7}$, \\ H. Schleicher ${ }^{8}$, W. Schmidt ${ }^{8}$, F. Schreier ${ }^{9}$, and R. Titz ${ }^{6}$
}

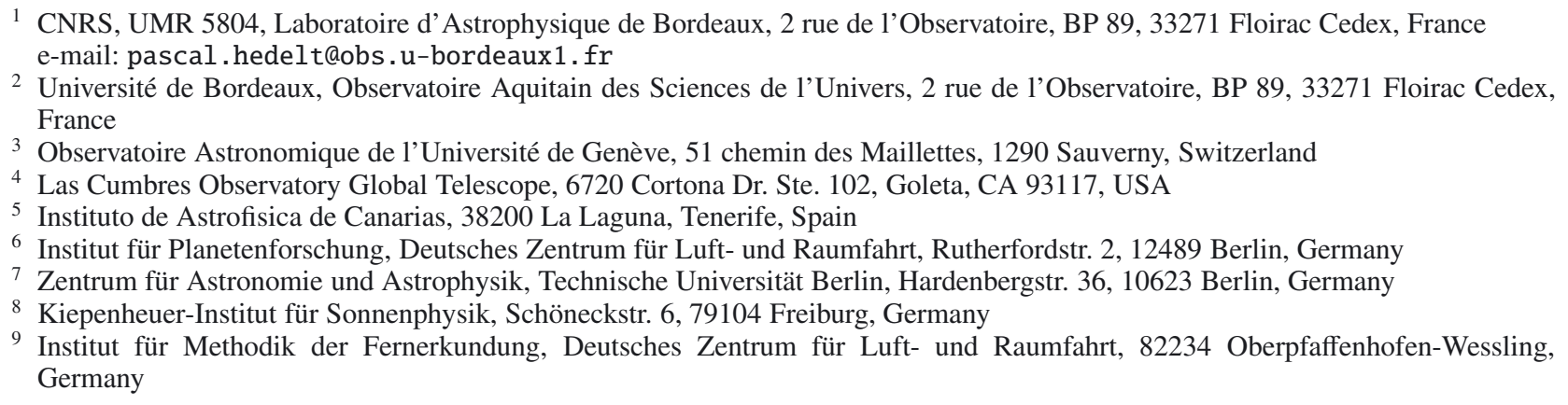

Received 30 November 2010 / Accepted 1 August 2011

\section{ABSTRACT}

\begin{abstract}
The transit of Venus in 2004 offered the rare possibility to remotely sense a well-known planetary atmosphere using ground-based absorption spectroscopy. Transmission spectra of Venus' atmosphere were obtained in the near infrared using the Vacuum Tower Telescope (VTT) in Tenerife. Since the instrument was designed to measure the very bright photosphere of the Sun, extracting Venus' atmosphere was challenging. We were able to identify $\mathrm{CO}_{2}$ absorption lines in the upper Venus atmosphere. Moreover, the relative abundance of the three most abundant $\mathrm{CO}_{2}$ isotopologues could be determined. The observations resolved Venus' limb, showing Doppler-shifted absorption lines that are probably caused by high-altitude winds. We demonstrate the utility of groundbased measurements in analyzing the atmospheric constituents of a terrestrial planet atmosphere using methods that might be applied in future to terrestrial extrasolar planets.
\end{abstract}

Key words. planets and satellites: atmospheres - planets and satellites: composition - planets and satellites: detection radiative transfer - techniques: spectroscopic

\section{Introduction}

Transits of Venus, during which Venus crosses the line of sight between Earth and the Sun, come in pairs separated by eight years followed by long gaps of $\sim 121.5$ and $\sim 105.5$ years. Thus, this pattern repeats every 243 years. The last transit occurred in 2004, whereas the next one will be observable in 2012. Apart from the inner planets of the solar system, extrasolar planets also have a certain possibility to transit in front of their central star, offering the feasibility of investigating their atmosphere during their primary transit. Until now, 144 transiting extrasolar planets have been discovered among the more than 500 exoplanets that have been found in total ${ }^{1}$. Among these, seven transiting planets might be classified as terrestrial: CoRoT-7 b (4.80 $M_{\text {Earth }}$, Léger et al. 2009), GJ 1214 b (6.36 $M_{\text {Earth }}$, Charbonneau et al. 2009), as well as the recently detected planets Kepler-11 b, d, e, f (4.30, 6.10, 8.40, 2.30 $M_{\text {Earth }}$, respectively, Lissauer et al. 2011) and Kepler-10 b (4.54 $M_{\text {Earth }}$, Batalha et al. 2011).

During a planetary transit, the light emitted by the central star traverses the planetary atmosphere and is attenuated before it reaches the observer. An analysis of the extinction allows us

\footnotetext{
1 See J. Schneider's Extrasolar Planet Encyclopedia at http:// exoplanet. eu (status: August 2011).
}

to characterize, for example, the chemical composition of the atmosphere. The apparent planetary radius at which the tangential optical depth is below unity varies at different wavelengths and can hence be used to infer the height of the atmosphere (related to pressure and atmospheric structure) and the existence of cloud layers (see e.g., Seager \& Sasselov 2000; Brown 2001).

In the past, transits of Mercury have offered the opportunity to detect absorption features within the atmosphere using ground-based spectroscopic observations. For example, during the Mercury transit in 2003, neutral sodium in its exosphere was detected using this technique (Schleicher et al. 2004). Even the Earth can be investigated as a transiting planet during lunar eclipse observations, when we can study the absorption lines of major atmospheric constituents such as $\mathrm{CH}_{4}, \mathrm{H}_{2} \mathrm{O}, \mathrm{O}_{2}, \mathrm{O}_{3}$, and $\mathrm{CO}_{2}$ in reflected light from the Sun by the Moon (see e.g., Pallé et al. 2009; Vidal-Madjar et al. 2010). Furthermore, Earthshine observations, where the reflected sunlight from Earth is observed on the Moon's night-side, show absorption signatures of $\mathrm{O}_{2}, \mathrm{O}_{3}$, and $\mathrm{H}_{2} \mathrm{O}$ as well as a signature of Earth's vegetation red edge (Arnold et al. 2002; Arnold 2008).

This technique can of course also be applied to transiting exoplanets. Molecular absorption bands in the infrared have been detected in the atmosphere of planets around bright stars (e.g., Knutson 2007; Tinetti et al. 2007; Swain et al. 2008). 
Furthermore, the sodium resonance doublet at $598.3 \mathrm{~nm}$ in the atmosphere of the transiting extrasolar giant planet HD $209458 \mathrm{~b}$ (Charbonneau et al. 2002) has been detected during four planetary transits, as well as neutral hydrogen (HI) (Vidal-Madjar et al. 2003), oxygen (OI), and carbon (CII) (Vidal-Madjar et al. 2004). Snellen et al. (2010) used high-resolution groundbased spectroscopy to detect CO lines in the atmosphere of HD $209458 \mathrm{~b}$ at about $2300 \mathrm{~nm}$. These lines were blue shifted by about $2 \mathrm{~km} \mathrm{~s}^{-1}$ with respect to the velocity of the host star, perhaps representing a velocity flow from the day to the night-side, which is also known as subsolar-to-antisolar (SSAS) flow.

However, performing transmission spectroscopy of terrestrial exoplanets and detecting the faint atmospheric absorption lines is a difficult task. Up to now, only one terrestrial exoplanet has been characterized spectroscopically using transmission spectroscopy: Bean et al. (2010) obtained transmission spectra from the terrestrial exoplanet GJ $1214 \mathrm{~b}$ in the range from 780 to $1000 \mathrm{~nm}$. These authors detected a lack of spectral features, which is indicative of either a dense, water-vapordominated atmosphere or a hydrogen-dominated atmosphere with a cloud layer that is opaque in the observed wavelength range. Additional observations by Croll et al. (2011) and Désert et al. (2011) rule out a fully water-dominated atmosphere. A solar-composition cloudy atmosphere without methane provides the closest description of the data (Miller-Ricci Kempton et al. 2011).

Major synthetic modeling of the spectra of hypothetical terrestrial exoplanets were performed by Des Marais et al. (2002), Segura et al. (2003, 2005), Tinetti (2006), Ehrenreich et al. (2006), Kaltenegger et al. (2007), Kaltenegger \& Traub (2009) and Rauer et al. (2011), in which the terrestrial planets of the solar system (i.e. Venus, Mars, and Earth) were investigated as if they were distant exoplanets. These studies also considered the effects of varying different parameters, such as the central star, the orbital distance, or the atmospheric composition. Ehrenreich et al. (2006) considered the appearance of Venus if it were seen as a transiting exoplanet orbiting various main-sequence stars.

This paper investigates the feasibility of the transmission spectroscopy technique for analyzing the atmosphere of a terrestrial (exo-) planet, by considering ground-based high-resolution measurements during a Venus transit. A cloud-free radiative transfer model was used to compare these measurements with synthetic transmission spectra of Venus' upper atmosphere above the cloud top, assuming a pure $\mathrm{CO}_{2}$ atmosphere.

In Sect. 2, the observations and the data reduction are described and the extracted limb spectra are discussed. The radiative transfer model and the calculations are described in Sect. 3. In Sect. 4, a parameter variation is performed, before the final fit to measured data is performed in Sect. 5. Finally, the results are summarized in Sect. 6 and implications for extrasolar planets are discussed in Sect. 7.

\section{Observations and reduction}

The transit of Venus on June 8, 2004 was observed at the Vacuum Tower Telescope (VTT) of the Kiepenheuer Institut für Sonnenphysik (KIS) in Tenerife. The observations of the transit lasted from 06:30h (UT), when the Sun rose with Venus already in front of its disk, until 11:26h (UT) at fourth contact. The VTT is a $70 \mathrm{~cm}$ diameter telescope with a focal length of $46 \mathrm{~m}$, equipped with an echelle spectrograph. The spectrograph slit corresponds to a width of $0.35^{\prime \prime}$ and a length of $92^{\prime \prime}$ on the sky. In the focal plane of the spectrograph, the Tenerife Infrared Polarimeter (TIP, Collados et al. 1999) detector was installed. It consists of four $128 \times 128$ pixel CCDs and was used in its spectrograph mode (without polarimeter). The spectral resolution of the entire system was $R=\lambda / \Delta \lambda=200000$. The image scale in the focal plane was $0.35^{\prime \prime} \mathrm{px}^{-1}$, thus one pixel corresponded to about $71 \mathrm{~km}$ at the position of Venus.

Three wavelength regions (identified by the observers) with strong absorption lines of $\mathrm{CO}_{2}$ were observed with a sampling of $0.031 \AA \mathrm{px}^{-1}$ :

\section{- 1596.49 nm-1597.29 nm ("Favorite") (1278 images); \\ - $1597.49 \mathrm{~nm}-1598.27 \mathrm{~nm}$ ("Favorite+") (4779 images); \\ - 1612.39 nm-1613.19 nm ("Grabbag") (4103 images).}

One single image is an integration of 20 exposures with an exposure time of $50 \mathrm{~ms}$ to provide $1 \mathrm{~s}$ integration time. Under the same conditions, 207 flatfield images of the unobscured solar disk and 127 darks were taken.

The telescope tracked the Sun and drift scans over the Venus disk were performed, i.e. Venus moved across the slit, which remained fixed with respect to the solar image. For the observations in the "Favorite" wavelength range, the slit was oriented at an angle of $315.5^{\circ}$ (measured from Venus' north pole, anticlockwise). During the observations in the "Grabbag", as well as in the "Favorite+" wavelength range, the slit was rotated to an angle of $45.5^{\circ}$ (measured from the Venusian north, anticlockwise) and the observation was then performed at this position.

We note that Alonso (2006) examined the same data with respect to latitudinal variations in absorption line positions, observing different depths of the spectral features in the "Favorite+" region at higher and lower latitudes, but without further interpretation of the data. For this paper an average over the different latitudes was instead used and the absorption of the three most abundant isotopologues of $\mathrm{CO}_{2}$ was thus investigated with a higher signal-to-noise ratio (SNR).

We note that the "Grabbag" region finally turned out not to be usable owing to a strong noise pattern and a strong gain jump between the right and left CCDs, which could not be corrected. Therefore, transmission spectra of this region are omitted in the following.

An example of a raw image (of $1 \mathrm{~s}$ integration time) is shown in Fig. 1a. It shows a ring-like fringe pattern, a central horizontal line separating the two upper $128 \times 128$ pixel CCDs from the lower ones, and a region with a noticeable number of bad pixels in the upper left corner. In this figure, the $y$-axis represents the position, whereas the $x$-axis is the wavelength. Slightly dark vertical bands correspond to the solar and terrestrial absorption lines. The disk of Venus can be seen as a thick horizontal band at the center of the image.

A standard image reduction procedure was used to calibrate the images, including dark current removal and flatfielding. We note that the final flat-field master image did not contain any absorption lines of either the Sun or Earth, but only the fringe pattern. Thus, in the final reduced images (see Fig. 1b), only the interference fringes are removed, whereas solar and telluric absorption lines are now clearly visible, as well as the separation between the CCDs.

The next step was to extract the spectra of Venus' atmosphere. First, the positions of both of Venus' limbs were identified on the reduced images and taken as reference points. The limb positions were determined by fitting the peaks of the first derivative of the spatial intensity with a Gaussian.

The mean diameter (taking into account the pixel scale of the $\mathrm{CCD}$ ) of the projected Venus disk in the "Favorite" region was 

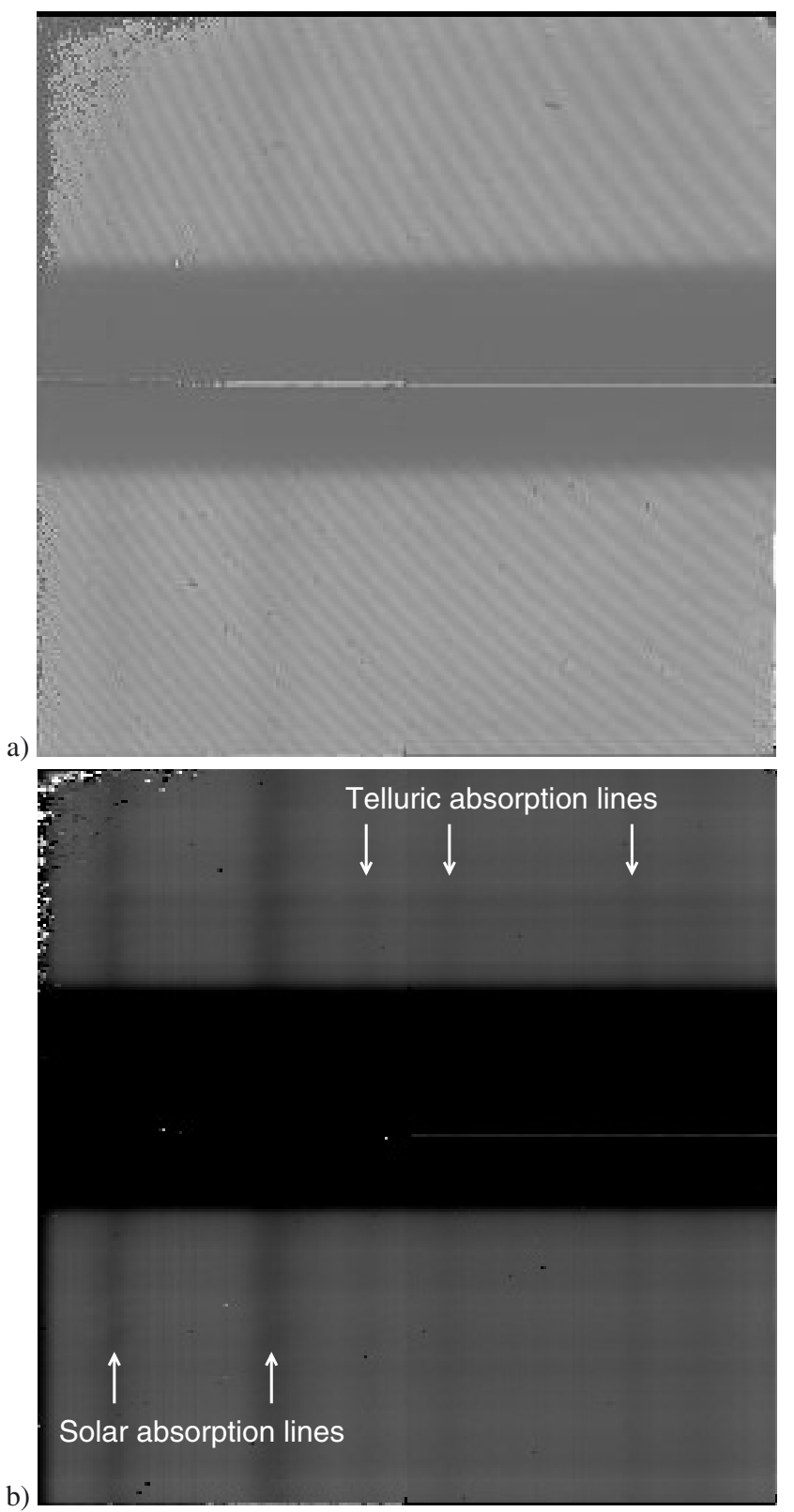

Fig. 1. a) Raw image from the "Favorite+" region: the Venus disk is clearly visible as the horizontal dark strip at the center of the image, in addition to the fringe pattern (sloping dark lines), the bad pixel area in the upper left corner, and the gain jump between the upper and lower CCD chips at the center of the frame. Strong solar and weak telluric absorption lines appear as vertical dark stripes. b) Reduced image, after flatfielding. Clearly visible are the solar and telluric absorption lines and the gain jump between the CCDs.

$7980.018 \pm 2164.985 \mathrm{~km}$, whereas in the "Favorite+" region it was $4683.8790 \pm 971.466 \mathrm{~km}$. Thus, the slit was never positioned over the center of the Venus disk, but more toward the limb, and the lower limit to the projected atmospheric altitude covered by one pixel is therefore $46.313 \pm 12.565 \mathrm{~km}$ in the "Favorite" region and $27.184 \pm 5.638 \mathrm{~km}$ in the "Favorite+" region, assuming that the radius of the visible disk of Venus is $6116.80 \mathrm{~km}$ (so as to include the opaque cloud deck at $65 \mathrm{~km}$ altitude). Therefore, the upper atmosphere can be resolved to within a few pixels.

Following this, a spectral and spatial normalization was performed. First, each image row was divided by a solar disk spectrum, which had been obtained by binning two image rows

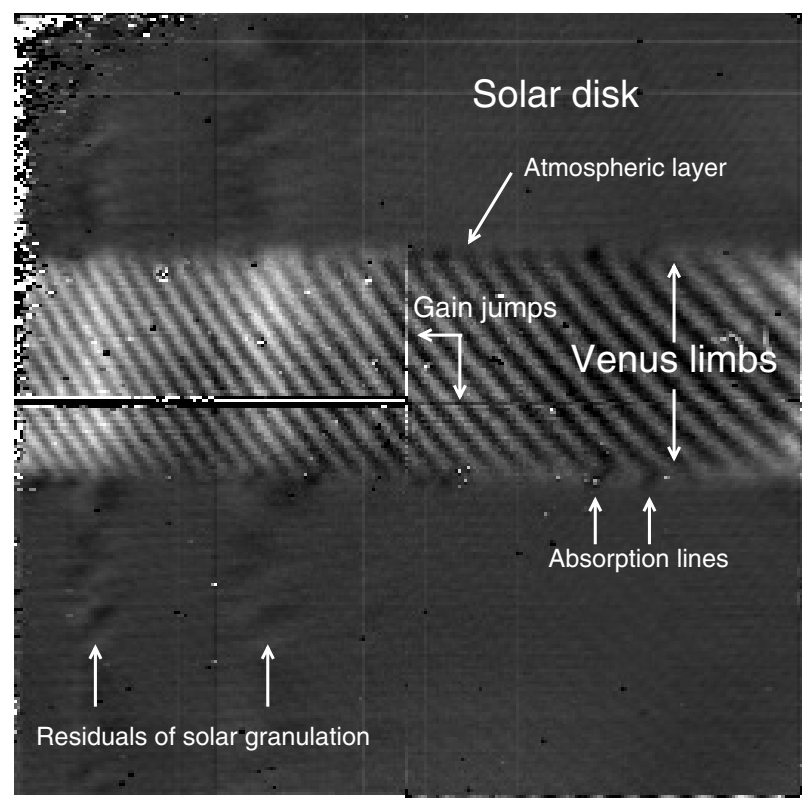

Fig. 2. Flatfielded and normalized image from the "Favorite+" region. In the transition region between the solar surface and the disk of Venus, a thin layer with absorption features is visible, which represents the atmospheric layers of Venus.

11 pixels offset from the Venus limb. This step removed the telluric absorption lines but only attenuated the solar absorption lines. To distinguish between telluric absorption lines and lines forming in the atmosphere of an exoplanet, ground-based observations of transiting exoplanets may take advantage of the Doppler shift due to the proper motions of the star, the exoplanet, the Sun, and the Earth (see e.g., Vidal-Madjar et al. 2010).

The spatial normalization was then performed by averaging image columns in a clean part of the CCD and dividing the original image by this averaged spectrum. The resulting image in Fig. 2 clearly shows in the transition region between the Venusian and solar disk weak absorption lines, which do not correlate with the solar absorption lines. We compared with the HITRAN2004 (High-Resolution Transmission Molecular Absorption Database, Rothman et al. 2005) line database to help us identify the atmospheric absorption lines as those of $\mathrm{CO}_{2}$. The flatfielding did not work properly at the position of the Venus disk, as the fringe pattern is still visible.

The residuals of solar absorption lines visible in Fig. 2 are caused by Doppler shifts of convecting granules in the photosphere of the Sun and the so-called "five-minute" oscillation. The Doppler shift of these residuals is $\sim 0.0143 \mathrm{~nm}$, which corresponds to a velocity of $1.7 \mathrm{~km} \mathrm{~s}^{-1}$ and a size of $\sim 1300 \mathrm{~km}$. These values are consistent with values measured by Carroll \& Ostlie (1996).

Finally, the spectra of Venus' atmosphere at the limb position were extracted and all spectra of a particular wavelength region were averaged, to improve the SNR. Thus, the information about the altitude and the corresponding Doppler-shifts caused by winds are lost. We note that the aforementioned normalization is inaccurate, primarily because of varying gain jumps between the CCDs at wavelengths with strong solar absorption lines that results in a distorted baseline in the averaged spectrum at each limb. The baseline in every image is therefore corrected by applying a ninth degree polynomial fit. We verified that the relative absorption depths were unaffected by this correction. Furthermore, in some images the atmospheric layers appear to be 

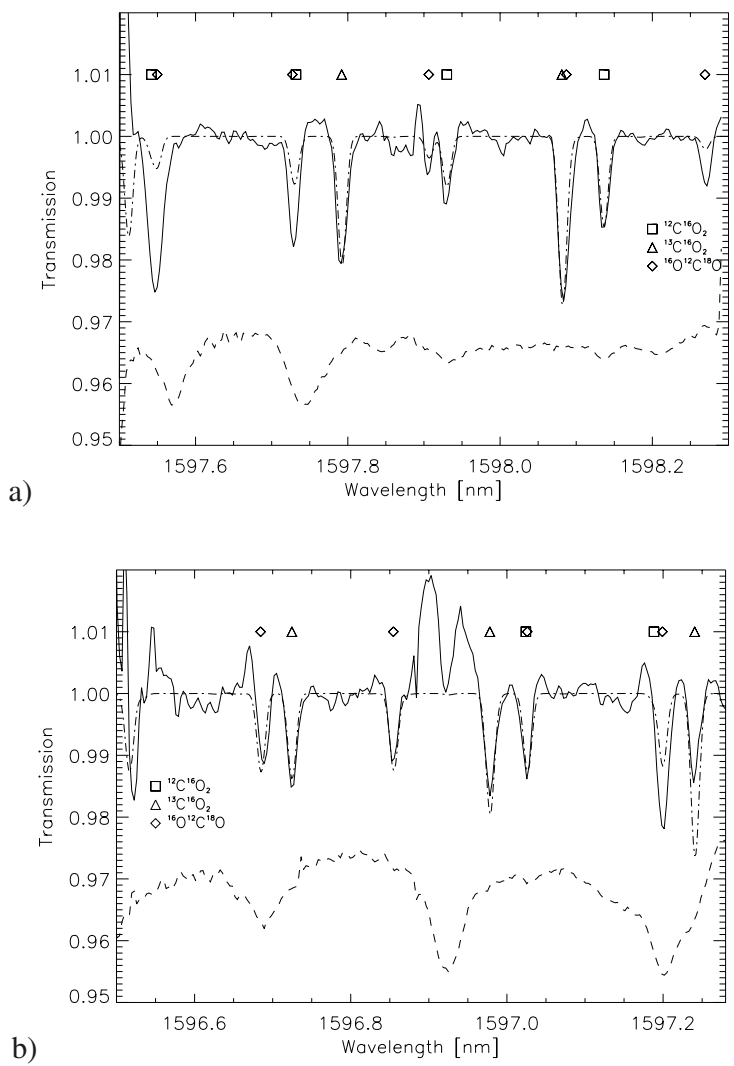

Fig. 3. Averaged spectrum (solid line) of both Venus limbs in the a) "Favorite+" and b) "Favorite" region together with the best-fit model spectrum (dash-dotted line, see text for details). Absorption line positions of the three isotopologues are indicated by different symbols. The dashed line shows a scaled spectrum of the Sun in arbitrary units, showing partial overlap of solar absorption lines with absorption lines from Venus' atmosphere.

disturbed by the fringe pattern within the Venus disk, inhibiting any detection of absorption lines. These images were removed manually before averaging all spectra.

In total, 395 images from the "Favorite" and 1107 images from the "Favorite+" region have been averaged. Using line positions from HITRAN as a reference, an accurate wavelength calibration was performed. In Figs. $3 a$ and $b$ we present the averaged spectra from the "Favorite+" and "Favorite" wavelength ranges, respectively. We note that the Doppler shift of absorption lines caused by the high wind velocities in the upper atmosphere of Venus was neglected, although superrotation plays a major role in the upper atmosphere dynamics.

However, a Doppler shift between adjacent pixel rows can still be measured. We note that the Venus absorption lines in Fig. 2 are tilted on both sides of the limb, with a blue shift of the lines being clear with increasing altitude on the eastern Venus limb (lower part of the Venus disk in Fig. 2), and a red shift of the absorption lines on the western limb. The Doppler-shifted lines yield a velocity gradient of about $7 \mathrm{~m} \mathrm{~s}^{-1}$ per $\mathrm{km}$ altitude, that remains nearly constant with increasing altitude on the western limb, while decreasing slightly on the eastern limb.

This Doppler shift might be caused by the combined effect of the superrotation of Venus' mesosphere and the SSAS flow in the upper atmosphere (Bougher et al. 1997). The SSAS wind velocities measured by Goldstein et al. (1991) and Betz et al. (1977) at an altitude of $100 \mathrm{~km}$ are in the range from 105 to $140 \mathrm{~m} \mathrm{~s}^{-1}$, whereas Lellouch et al. (2008) analyzed data from the Venus Express mission measuring wind speeds in the range from 30 to $50 \mathrm{~m} \mathrm{~s}^{-1}$ at $\sim 93 \mathrm{~km}$ altitude that strongly increased to between 90 and $120 \mathrm{~m} \mathrm{~s}^{-1}$ at $\sim 102 \mathrm{~km}$ altitude. The velocity gradient measured from the Venus transit spectra thus agrees with the gradient determined by Lellouch et al. (2008). A detailed study of this effect is however beyond the scope of this paper and will be the subject of a following paper, perhaps incorporating observations during the next Venus transit in June 2012.

\section{Radiative transfer calculations}

The radiative transfer model SQuIRRL (Schwarzschild Quadrature InfraRed Radiation Line-by-line, Schreier \& Schimpf 2001) was used to fit the measured spectra. SQuIRRL is a cloud free, line-by-line radiative transfer model that uses the molecular absorption-line database HITRAN to calculate absorption cross-sections. It generates emission and absorption spectra for arbitrary atmospheric paths, assuming local thermodynamic equilibrium (LTE). Furthermore, a convolution with appropriate spatial and spectral instrumental response functions can be performed.

The model atmosphere extends from $0 \mathrm{~km}$ to $180 \mathrm{~km}$ altitude, divided into 30 layers. We assume that $\mathrm{CO}_{2}$ is the only atmospheric absorber with an isoprofile of $96.5 \%$ abundance up to an altitude of $100 \mathrm{~km}$. At greater altitudes, the $\mathrm{CO}_{2}$ profile from Hedin et al. (1983) was used. For the transmission calculations, below an altitude of $100 \mathrm{~km}$, we adopted the day-side temperature profile from Seiff et al. (1985), whereas above we adopted the profile from Hedin et al. (1983), which agrees with solar occultation measurements of Venus' mesosphere from the Venus Express mission (see e.g., Mahieux et al. 2010).

Transmission spectra for a set of different tangential heights above $100 \mathrm{~km}$ altitude are calculated for each wavelength region, using a Gaussian beam of $24 \mathrm{~km}$ and $15 \mathrm{~km}$ half-width halfmaximum $(H W H M)$ for the "Favorite" and "Favorite+" range, respectively, according to the average projected atmospheric height in the respective wavelength region. We note that although one pixel corresponds to about $71 \mathrm{~km}$ at the position of Venus as mentioned in Sect. 2, the slit was not positioned perpendicular to the surface, but at a certain angle since the spectra were taken close to the limb of Venus' disk. Thus, the projected atmospheric height is used to account for the field of view. The resulting spectra are finally convolved with a Gaussian of $6.15 \times 10^{-3} \mathrm{~nm} H W H M$ to account for the spectral resolution of the instrument.

We note that we include no clouds or aerosol opacity in the radiative transfer model. Nevertheless, the observed spectra can be reproduced quite well because the best-fitting tangential heights of the extracted spectra are well above an altitude of $100 \mathrm{~km}$, as we later show. Above $100 \mathrm{~km}$, clouds can be neglected to a good approximation because extinction by $\mathrm{H}_{2} \mathrm{SO}_{4}$ cloud particles is expected to extend only up to about $80 \mathrm{~km}$ altitude (see e.g., Esposito et al. 1983; Roos et al. 1993; or Grinspoon et al. 1993). The analysis of Venus Express SPICAV/SOIR observations acquired by Wilquet et al. (2009) demonstrated that the extinction coefficient of the atmospheric haze measured at $1553.7 \mathrm{~nm}$ decreases by about two orders of magnitude to about $2 \times 10^{-4} \mathrm{~km}^{-1}$ at $90 \mathrm{~km}$, which is by far lower than the $\mathrm{CO}_{2}$ absorption coefficient and can thus be neglected for the altitudes considered here.

Furthermore, the assumption of LTE for the radiative transfer calculations may not hold in the altitude range considered in this paper. Both Roldán et al. (2000) and 


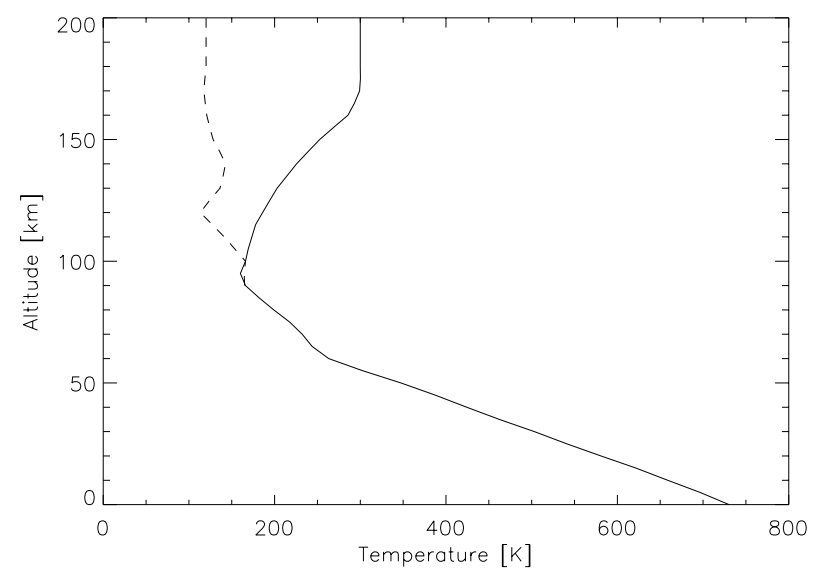

Fig. 4. Temperature profiles used for the calculation of transmission spectra, obtained from Yung \& DeMore (1999). Solid line: day-side, Dotted line: night-side.

López-Valverde et al. (2007) found a departure from LTE conditions above $90 \mathrm{~km}$ altitude (depending on the $\mathrm{CO}_{2}$ band) that produced rather strong emission lines. Nevertheless, non-LTE conditions in the wings of the $1600 \mathrm{~nm}$ absorption band, as considered in this paper, can be neglected for several reasons:

- Under non-LTE conditions, the excitation temperature of the $\mathrm{CO}_{2}(30011,30012,30013)$ levels (which correspond to the observed absorption lines) related to solar excitation is about $400 \mathrm{~K}$ at altitudes above about $120 \mathrm{~km}$ in the wavelength range considered here (López-Puertas, priv. comm.). This temperature is higher than the atmospheric temperature of about $300 \mathrm{~K}$ under LTE conditions (see Fig. 4). However, the solar flux with a blackbody temperature of about $5800 \mathrm{~K}$ is much higher than the flux emitted in the Venus atmosphere.

- López-Valverde et al. (2007) found only a very weak atmospheric emission (which is below the detection limit of the VIRTIS instrument on Venus Express) for the $1600 \mathrm{~nm}$ $\mathrm{CO}_{2}$ band at the limb for low solar zenith angles (i.e. for the transmission geometry considered in this paper), which is even lower in the band wings where the Venus transit observations were performed.

- Potential non-LTE effects caused by the non-LTE populations of the lower vibrational level of hot bands can also be neglected, since the observed lines correspond to a fundamental transition from the ground vibrational level.

To summarize, the contribution of the non-LTE atmospheric emission is in any case much smaller than the Sun's radiation, thus strong lines seen in emission are not expected in the considered wavelength range. Any influence on the absorption can be also neglected. We note that observations of the next Venus transit in 2012 may provide data of much higher quality and at wavelengths where non-LTE effects cannot be neglected. In that case, a more detailed radiative-transfer code needs to be used, which includes non-LTE effects as well as cloud and aerosol extinction.

For a transiting exoplanet, we note that the temperature profile is unavailable. The day-side temperature profile might be inferred from atmospheric modeling and secondary eclipse observations by investigating the emission spectrum. However, for transmission observations the terminator temperature profile cannot be easily inferred, since it depends on numerous factors, such as planetary rotation, atmospheric composition and dynamics, and distance to star. Nevertheless, transmission observations

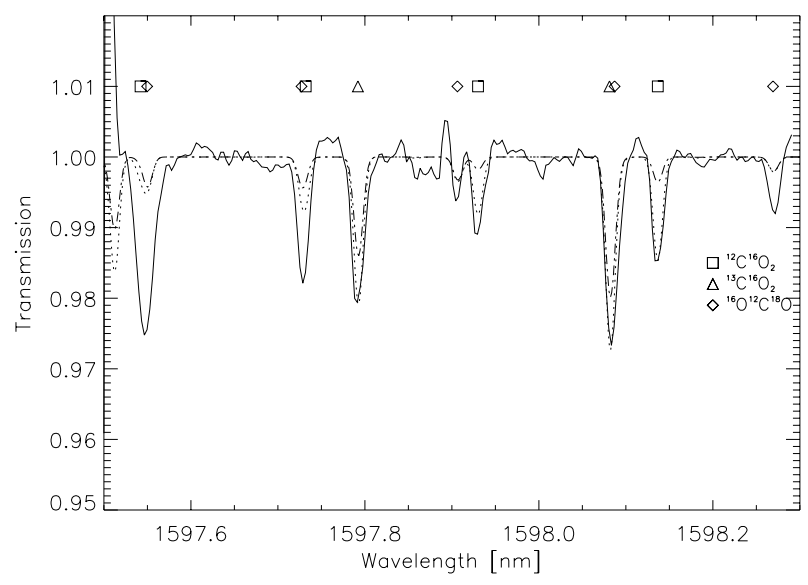

Fig. 5. Spectral response when varying atmospheric temperature profile. Solid line: measured spectrum from the "Favorite+" region, dotted line: spectrum calculated with the day-side temperatures profile and dash dotted line: spectrum calculated with the night-side temperature profile. Absorption line positions of the three isotopologues are indicated by different symbols.

of the hot-Jupiter planet HD 209458 b by Sing et al. (2008) and Vidal-Madjar et al. (2011) were able to infer a temperature profile at the terminator by investigating sodium absorption-line profiles.

Close-in exoplanets that may have a thick atmosphere and rotate slowly will be more likely to have a strong SSAS flow to transport warm air from their hot day-side to the cold night-side, thus a day-side temperature profile might be applicable for the terminator region. Furthermore, these atmospheres will have a higher atmospheric scale-height on the day-side (see for example Burrows et al. 2010, showing this effect on a transiting giant exoplanet), which could in principle be detected by determining the radius during the ingress and egress. However, this effect is expected to be quite small.

\section{Parameter variation}

To determine the best-fit model parameters, a parameter variation was performed to investigate the temperature and height dependence of the absorption lines. Furthermore, the abundances of the three isotopologues found in the data was varied to infer isotopic ratios for ${ }^{12} \mathrm{C} /{ }^{13} \mathrm{C}$ and ${ }^{16} \mathrm{O} /{ }^{18} \mathrm{O}$ in Venus' atmosphere.

Although a fixed temperature profile was used for the final fit, the profile was varied within the temperatures of the day and night-side to quantify the response of the absorption. In particular, for the upper atmospheric layers above $100 \mathrm{~km}$, large differences in temperature between the day and night-side of Venus occur (see Fig. 4), reaching about $150 \mathrm{~K}$ above altitudes of $170 \mathrm{~km}$. Increasing the temperature from the night to the dayside profile, the absorption of ${ }^{12} \mathrm{C}^{16} \mathrm{O}_{2}$ and ${ }^{13} \mathrm{C}^{16} \mathrm{O}_{2}$ increases by about $0.5 \%$ as can be seen in Fig. 5. The temperature variation has no significant effect on ${ }^{12} \mathrm{C}^{16} \mathrm{O}^{18} \mathrm{O}$.

The tangential height of the beam was varied in steps of $1 \mathrm{~km}$ in the altitude range from $100 \mathrm{~km}$ to $200 \mathrm{~km}$. With an increase of $1 \mathrm{~km}$ in tangential height, the absorption of all lines was found to decrease by about $1.3 \%$. Since ${ }^{12} \mathrm{C}^{16} \mathrm{O}_{2}$ is the most abundant isotopologue and hence independent of the isotopic ratio, lines of this isotopologue were used to determine the best-fit tangential height of the measurements. We note that the determination of the tangential height of course also depends on the chosen beam width. 
Table 1. Carbon and oxygen isotopic ratios.

\begin{tabular}{lccc}
\hline \hline Planet & ${ }^{12} \mathrm{C} /{ }^{13} \mathrm{C}$ & ${ }^{16} \mathrm{O} /{ }^{18} \mathrm{O}$ & Reference \\
\hline Earth & $89.01 \pm 0.38$ & $498.71 \pm 0.25$ & $(1)$ \\
Venus & $86 \pm 12$ & $500 \pm 80$ & $(2)$ \\
Venus & $110 \pm 30$ & $537 \pm 53$ & $(3)$ \\
\hline
\end{tabular}

References. (1) Yung \& DeMore 1999; (2) Bezard et al. 1987; (3) This work

Using initially Venus isotopic ratios of ${ }^{12} \mathrm{C} /{ }^{13} \mathrm{C}$ and ${ }^{16} \mathrm{O} /{ }^{18} \mathrm{O}$ (see also Table 1), isotopic ratios were varied by large amounts, i.e. in the range from half up to double isotopic ratios of Venus. The abundances of the $\mathrm{CO}_{2}$ isotopologues can be calculated according to the relationship from Bezard et al. (1987)

${ }^{12} \mathrm{C} /{ }^{13} \mathrm{C}={ }^{12} \mathrm{CO}_{2} /{ }^{13} \mathrm{CO}_{2}$

${ }^{16} \mathrm{O} /{ }^{18} \mathrm{O}=2{ }^{12} \mathrm{C}^{16} \mathrm{O}_{2} /{ }^{12} \mathrm{C}{ }^{16} \mathrm{O}{ }^{18} \mathrm{O}$.

It can be clearly seen that increasing the isotopic ratios, the abundances of ${ }^{13} \mathrm{C}^{16} \mathrm{O}_{2}$ and ${ }^{12} \mathrm{C}^{16} \mathrm{O}^{18} \mathrm{O}$ decrease.

\section{Spectral fitting}

The fitting of both calculated and measured data was performed visually because it was impossible to use a least squares fit owing to the poor spectral normalization and the remaining solar residuals and noise features. In the fitting, we used only isolated lines with a good baseline that did not overlap with solar absorption lines.

\section{1. "Favorite+" wavelength range}

In the "Favorite+" region (Fig. 3a), the ${ }^{12} \mathrm{C}^{16} \mathrm{O}_{2}$ absorption lines at both $1597.93 \mathrm{~nm}$ and $1598.14 \mathrm{~nm}$ (box symbols in the figure) can be used in the tangential height determination. The absorption strength may however be disturbed by the higher baseline nearby. Using these lines, the closest fit was achieved for a tangential height of $116 \pm 1 \mathrm{~km}$ and a beam width of $15 \mathrm{~km} \mathrm{HWHM}$. After taking into account the uncertainty in the beam width (see Sect. 2), we found that the best-fit tangential height is $116 \pm 5 \mathrm{~km}$. All other lines of this isotopologue overlap with other isotopologues or are at positions where solar absorption lines are located. We note that the inferred tangential height is close to the homopause located at about an altitude of $130 \mathrm{~km}$, above which turbulent mixing of the atmosphere becomes unimportant and the atmosphere becomes stratified. Although this result could influence the isotopic ratio over the altitude region probed, the atmospheric levels above the inferred tangential height do not contribute much to the absorption lines, hence any influence is neglected. Furthermore, the data quality is too low as to resolve this effect.

The absorption lines of ${ }^{13} \mathrm{C}^{16} \mathrm{O}_{2}$ at $1597.79 \mathrm{~nm}$ and at $1598.08 \mathrm{~nm}$ (triangle symbols in the figure) are used to determine the carbon isotopic ratio, giving a best fit of ${ }^{12} \mathrm{C} /{ }^{13} \mathrm{C}=$ $110 \pm 30$, which is higher than the value for Venus inferred from Bezard et al. (1987). However, taking into account the deviated baseline, the values are within the error margin comparable to literature values. Although the line at $1598.08 \mathrm{~nm}$ overlaps with ${ }^{12} \mathrm{C}^{16} \mathrm{O}{ }^{18} \mathrm{O}$, the absorption is dominated by ${ }^{13} \mathrm{C}^{16} \mathrm{O}_{2}$.

The isolated lines of ${ }^{12} \mathrm{C}^{16} \mathrm{O}^{18} \mathrm{O}$ at $1597.91 \mathrm{~nm}$ and $1598.27 \mathrm{~nm}$ (diamond symbols in the figure) could not be used to determine the isotopic ratio of ${ }^{16} \mathrm{O} /{ }^{18} \mathrm{O}$ owing to the spike at about $1597.89 \mathrm{~nm}$, although varying the ${ }^{16} \mathrm{O} /{ }^{18} \mathrm{O}$ ratio has only a marginal effect on the absorption. Therefore, we also considered the isotopic ratio found in the "Favorite" region (see hereafter) where ${ }^{16} \mathrm{O} /{ }^{18} \mathrm{O}=537 \pm 53$.

Figure 3 a compares the measured spectrum with the bestfit model spectrum for a tangential height of $116 \mathrm{~km}$, and isotopic ratios of ${ }^{12} \mathrm{C} /{ }^{13} \mathrm{C}=110$ and ${ }^{16} \mathrm{O} /{ }^{18} \mathrm{O}=537$. In addition, a scaled solar spectrum extracted from a flat-fielded image of the "Favorite+" region is plotted (dashed) to visualize the location of the solar absorption lines.

\section{2. "Favorite" wavelength range}

In the "Favorite" region (Fig. 3b), the lines of ${ }^{12} \mathrm{C}^{16} \mathrm{O}_{2}$ are very weak and masked by absorption lines of the other isotopologues and no isolated absorption lines can be found. To determine the best-fit tangential height, absorption lines of ${ }^{13} \mathrm{C}^{16} \mathrm{O}_{2}$ are used, assuming that the isotopic ratio of ${ }^{12} \mathrm{C} /{ }^{13} \mathrm{C}$ is the same as in the "Favorite+" region. Absorption lines at $1596.73 \mathrm{~nm}$ and $1596.98 \mathrm{~nm}$ provide a best-fit tangential height of $120 \pm 2 \mathrm{~km}$, when using a beam width of $24 \mathrm{~km} H W H M$. Taking into account the uncertainty in the beam width, the estimated tangential height is $120 \pm 10 \mathrm{~km}$.

To help us determine the ${ }^{16} \mathrm{O} /{ }^{18} \mathrm{O}$ ratio, we selected the absorption lines of ${ }^{12} \mathrm{C}^{16} \mathrm{O}{ }^{18} \mathrm{O}$ at $1596.69 \mathrm{~nm}, 1596.85 \mathrm{~nm}$, and $1597.03 \mathrm{~nm}$. The first two lines were most closely fitted with ${ }^{16} \mathrm{O} /{ }^{18} \mathrm{O}=575$, whereas the other line was most closely fitted with ${ }^{16} \mathrm{O} /{ }^{18} \mathrm{O}=500$. We note that the line at $1596.69 \mathrm{~nm}$ may be influenced by the strong baseline deviation shortward of this line and the overlap with a solar line. Therefore, the estimated best-fit isotopic ratio is ${ }^{16} \mathrm{O} /{ }^{18} \mathrm{O}=537 \pm 53$. This is also higher than the value determined by Bezard et al. (1987), but still in the range of their uncertainty.

Figure $3 \mathrm{~b}$ shows the measured spectrum together with the best-fit model spectrum with a tangential height of $120 \mathrm{~km}$, and isotopic ratios of ${ }^{12} \mathrm{C} /{ }^{13} \mathrm{C}=110$ and ${ }^{16} \mathrm{O} /{ }^{18} \mathrm{O}=537$. As in the "Favorite+" region, a scaled solar spectrum is plotted (dashed) to visualize the location of the solar absorption lines.

\section{Summary and conclusion}

Using the Tenerife Infrared Polarimeter at the Vacuum Tower Telescope (VTT) in Tenerife, we have detected the molecular absorption lines of the three most abundant $\mathrm{CO}_{2}$ isotopologues ${ }^{12} \mathrm{C}^{16} \mathrm{O}_{2},{ }^{13} \mathrm{C}^{16} \mathrm{O}_{2}$, and ${ }^{12} \mathrm{C}^{16} \mathrm{O}^{18} \mathrm{O}$ within the atmosphere of Venus during its transit in June 2004. Although extracting limb spectra from the measured images was challenging, we have been able to reproduce the spectra using a line-by-line radiative transfer model. This modeling allowed us to determine the isotopic ratios of ${ }^{12} \mathrm{C} /{ }^{13} \mathrm{C}$ and ${ }^{16} \mathrm{O} /{ }^{18} \mathrm{O}$, which are slightly higher, but still agree with literature values. We note that the determination of isotopic ratios does not provide an additional measurement to those already available for Venus, but rather illustrates what might be possible for exoplanets in the future. Furthermore, by varying the tangential height while leaving the temperature profile fixed, good agreement between modeled and measured spectra could be achieved with tangential heights in the range from $(116 \pm 5) \mathrm{km}$ to $(120 \pm 10) \mathrm{km}$, depending on the wavelength range considered.

The observations resolved Venus' limb, showing an increasing Doppler-shift of the absorption lines with increasing altitude. This is probably caused by high altitude winds in Venus' atmosphere, with a wind velocity gradient being consistent with the Venus Express measurements of Lellouch et al. (2008). 
The results illustrate the feasibility of the transmission spectroscopy technique to identify atmospheric constituents and even infer isotopic ratios in terrestrial exoplanets. Future planned space missions, which are designed to measure the atmospheres of terrestrial exoplanets, will utilize similar techniques to examine their atmospheres in detail.

\section{Implications for extrasolar planets}

Current models for exoplanets mostly rely on knowledge derived from the solar system planets. It is straightforward (at least conceptually) to generate a spectrum for a given planet type. The inverse problem, however, of inferring the characteristics of a planet from a spectrum is much more difficult owing to its illposed nature.

Transiting exoplanets cannot be resolved in front of their central star as in the case of the Venus transit presented here. Thus, the method to obtain atmospheric transmission spectra of an exoplanet therefore is different from that performed in this work. For transits of exoplanets, where the image of the planet and its central star are convolved by a few pixels, the total flux of the system has to be compared during and outside the transit. Since the apparent radius of the planet and thus the transit depth of the transiting planet depends on wavelength because of the absorbing atmosphere, a transmission spectrum can be obtained by observing the transit at different wavelengths.

Current and near-future space telescopes provide only low resolution spectroscopy (Spitzer: $R<600$, James Webb Space Telescope: $R<3000$ ), thus only molecular absorption bands can be investigated rather than single absorption lines. Although ground-based telescopes do operate with high-resolution spectrographs of $R \sim 100000$ such as the CRyogenic high-resolution InfraRed Echelle Spectrograph (CRIRES, Kaeufl et al. 2004) at the Very Large Telescope (VLT), the SNR is still too low to detect lines of different isotopologues as performed here, since the atmospheric absorption lines of exoplanets are far too faint relative to the overall stellar flux. Atmospheric features of exoplanets have a strength relative to the continuum of $10^{-6}$, which is much smaller than the 1-2\% absorption detected for Venus here. This makes the investigation of the atmosphere far more challenging. Furthermore, ground-based observations have to remove telluric absorption lines before accessing the atmospheric absorption of an extrasolar planet.

Nevertheless, the investigation of a terrestrial exoplanet is already possible, as has been demonstrated for GJ 1214b (Bean et al. 2010). The data can however be interpreted in several ways and the actual composition of the atmosphere remains unclear (see e.g., Croll et al. 2011; Désert et al. 2011; Miller-Ricci Kempton et al. 2011).

The composition of Venus and its $p, T$ profiles have been well-constrained by satellite and ground-based observations, which is of course not true for exoplanets. Temperature profiles of exoplanets might be inferred by emission spectroscopy during secondary eclipse observations. For example, $\mathrm{CO}_{2}$ absorption bands seen in emission would indicate a temperature inversion in the stratosphere since $\mathrm{CO}_{2}$ can be assumed to be well mixed throughout the atmosphere, as in the case of Venus, Mars, and Earth. In addition, the surface temperature might be inferred in spectral windows where the atmosphere is transparent down to the ground. The thermal emission of about 30 exoplanets has already been detected, allowing the determination of the temperature structure, energy redistribution, and day-night contrasts (see e.g., Deming et al. 2005; Harrington et al. 2006; Knutson 2007).
The measurement of exoplanet isotopic ratios (as shown in this paper for Venus) would provide insight into the evolution and diversity of exoplanet systems. However, the acquisition of data for this purpose is beyond the ability of current and nearfuture observational efforts, because both a significant SNR and a high spectral resolution are required to detect and separate the lines.

The Venus transit was observed with a spectral resolution of $R=200000$ in a wavelength range across which the three most abundant isotopologues are detectable. In the wavelength range observed, a resolution of more than about 25000 is required to separate lines of ${ }^{12} \mathrm{C}^{16} \mathrm{O}_{2}$ and ${ }^{13} \mathrm{C}^{16} \mathrm{O}_{2}$ and determine the ${ }^{12} \mathrm{C} /{ }^{13} \mathrm{C}$ ratio. To detect ${ }^{12} \mathrm{C}{ }^{16} \mathrm{O}{ }^{18} \mathrm{O}$ absorption lines and determine the ${ }^{16} \mathrm{O} /{ }^{18} \mathrm{O}$ ratio, a much higher spectral resolution of about $R=50000$ is required, to separate them from the ${ }^{12} \mathrm{C}^{16} \mathrm{O}_{2}$ and ${ }^{13} \mathrm{C}^{16} \mathrm{O}_{2}$ absorption lines. However, other molecules might display stronger absorption lines that are well separated.

For the transmission spectrum of an Earth-like planet around a solar-like G star at a distance of 10 pc, Rauer et al. (2011) demonstrated that a low spectral resolution of $R=2000$ could provide a photon-limited SNR of about 0.054 for the $4200 \mathrm{~nm}$ $\mathrm{CO}_{2}$ band, when using e.g., the James Webb Space Telescope (JWST), which has an aperture of $6.5 \mathrm{~m}$. At $R=10000$, the corresponding SNR would be 0.022 and at $R=50000$ it would be 0.009 , hence hundreds of transits need to be co-added in order to achieve a reasonable SNR of more than three. Since the SNR is directly proportional to the telescope aperture, a larger telescope aperture can provide data of a higher SNR. However, only the next generation telescopes with far larger apertures of about $40 \mathrm{~m}$ (e.g., the European Extremely Large Telescope, E-ELT) will provide SNRs close to unity for terrestrial exoplanets. Observations of close-in hot-Jupiter planets will also significantly increase the SNR. Thus, the determination of the isotopic ratios in an exoplanet atmosphere should be achievable in the near future.

Despite the difficulties related to the high-resolution spectroscopy of extrasolar planets, the observations of Venus shown here illustrate the potential of this analysis. Once sufficiently sensitive instrumentation becomes available, the search for isotopic lines in exoplanet atmospheres will be able to provide robust constraints on the atmospheric evolution of these distant planets.

Acknowledgements. We thank two anonymous referees for a thorough reading of and useful comments on the manuscript. We are grateful to Manuel López-Puertas for comments and discussion about possible non-LTE effects in the atmosphere of Venus. P.H. acknowledges support from the European Research Council (Starting Grant 209622: E 3 ARTHs). The VTT is operated by the Kiepenheuer Institut für Sonnenphysik at the Spanish Observatorio del Teide of the Instituto de Astrofísica de Canarias.

\section{References}

Alonso, R. 2006, Ph.D. Thesis, Universidad de La Laguna, available online at http://www.iac.es/galeria/ras/tesis/index.html

Arnold, L. 2008, Space Sci. Rev., 135, 323

Arnold, L., Gillet, S., Lardière, O., Riaud, P., \& Schneider, J. 2002, A\&A, 392, 231

Batalha, N. M., Borucki, W. J., Bryson, S. T., et al. 2011, ApJ, 729, 27

Bean, J. L., Kempton, E., \& Homeier, D. 2010, Nature, 468, 669

Betz, A. L., Sutton, E. C., McLaren, R. A., \& McAlary, C. W. 1977, in Planetary Atmospheres, ed. A. Vallance Jones, 29

Bezard, B., Baluteau, J. P., Marten, A., \& Coron, N. 1987, Icarus, 72, 623

Bougher, S. W., Alexander, M. J., \& Mayr, H. G. 1997, Venus II, ed. S. W. Bougher, D. M. Hunten, \& R. J. Phillips (Tucson: Univ. of Arizona Press), 259

Brown, T. M. 2001, ApJ, 553, 1006

Burrows, A., Rauscher, E., Spiegel, D. S., \& Menou, K. 2010, ApJ, 719, 341 
Carroll, B. W., \& Ostlie, D. A. 1996, An Introduction to Modern Astrophysics (Addison-Wesley Publishing Company, Inc.)

Charbonneau, D., Brown, T. M., Noyes, R. W., \& Gilliland, R. L. 2002, ApJ, 568,377

Charbonneau, D., Berta, Z. K., Irwin, J., et al. 2009, Nature, 462, 891

Collados, M., Hidalgo, R., Bellot Rubio, I. L., Ruiz Cobo, B., \& Soltau, D. 1999, in Abstracts of Contributed Talks and Posters presented at the Annual Science Meeting of the Astronomische Gesellschaft, Göttingen, Germany, 20-25 September 1999, AG Abstract Series, Vol. 15

Croll, B., Albert, L., Jayawardhana, R., et al. 2011, ApJ, 736, 78

Deming, D., Seager, S., Richardson, L. J., \& Harrington, J. 2005, Nature, 434, 740

Des Marais, D. J., Harwit, M. O., Jucks, K. W., et al. 2002, Astrobiol., 2, 153

Désert, J.-M., Bean, J., Miller-Ricci Kempton, E., et al. 2011, ApJ, 731, L40

Ehrenreich, D., Tinetti, G., Lecavelier Des Etangs, A., Vidal-Madjar, A., \& Selsis, F. 2006, A\&A, 448, 379

Esposito, L. W., Knollenberg, R. G., Marov, M. Y., Toon, O. B., \& Turco, R. 1983, in VENUS, ed. D. M. Hunten, L. Colin, T. M. Donahue, \& V. I. Moroz (The University of Arizona Press), 1, 484

Goldstein, J. J., Mumma, M. J., Kostiuk, T., et al. 1991, Icarus, 94, 45

Grinspoon, D. H., Pollack, J. B., Sitton, B. R., et al. 1993, Planet. Space Sci., 41, 515

Harrington, J., Hansen, B. M., Luszcz, S. H., et al. 2006, Science, 314, 623

Hedin, A. E., Niemann, H. B., Kasprzak, W. T., \& Seiff, A. 1983, J. Geophys. Res., 88, 73

Kaeufl, H., Ballester, P., Biereichel, P., et al. 2004, in SPIE Conf. Ser. 5492, ed. A. F. M. Moorwood, \& M. Iye, 1218

Kaltenegger, L., \& Traub, W. A. 2009, ApJ, 698, 519

Kaltenegger, L., Traub, W. A., \& Jucks, K. W. 2007, ApJ, 658, 598

Knutson, H. A. 2007, Nature, 448, 143

Léger, A., Rouan, D., Schneider, J., et al. 2009, A\&A, 506, 287

Lellouch, E., Paubert, G., Moreno, R., \& Moullet, A. 2008, Planet. Space Sci., 56,1355

Lissauer, J. J., Fabrycky, D. C., Ford, E. B., et al. 2011, Nature, 470, 53

López-Valverde, M. A., Drossart, P., Carlson, R., Mehlman, R., \& Roos-Serote, M. 2007, Planet. Space Sci., 55, 1757

Mahieux, A., Vandaele, A. C., Neefs, E., et al. 2010, J. Geophys. Res. (Planets), 115, E12014
Miller-Ricci Kempton, E., Zahnle, K., \& Fortney, J. J. 2011, ApJ, submitted [arXiv: 1104.5477]

Pallé, E., Zapatero Osorio, M. R., Barrena, R., Montañés-Rodríguez, P., \& Martín, E. L. 2009, Nature, 459, 814

Rauer, H., Gebauer, S., von Paris, P., et al. 2011, A\&A, 529, A8

Roldán, C., López-Valverde, M. A., López-Puertas, M., \& Edwards, D. P. 2000, Icarus, 147,11

Roos, M., Drossart, P., Encrenaz, T., et al. 1993, Planet. Space Sci., 41, 505

Rothman, L. S., Jacquemart, D., Barbe, A., et al. 2005, J. Quant. Spect. Rad. Trans., 96, 139

Schleicher, W., Wiedemann, G., Wöhl, H., Berkefeld, T., \& Soltau, D. 2004, A\&A, 425, 1119

Schreier, F., \& Schimpf, B. 2001, in International Radiation Symposium IRS2000: Current Problems in Atmospheric Radiation, ed. W. Smith, \& Y. Timofeyev (A. Deepak Publishing), 381

Seager, S., \& Sasselov, D. D. 2000, ApJ, 537, 916

Segura, A., Krelove, K., Kasting, J. F., et al. 2003, Astrobiol., 3, 689

Segura, A., Kasting, J. F., Meadows, V., et al. 2005, Astrobiol., 5, 706

Seiff, A., Schofield, J. T., Kliore, A. J., Taylor, F. W., \& Limaye, S. S. 1985, Adv. Space Res., 5, 3

Sing, D. K., Vidal-Madjar, A., Lecavelier des Etangs, A., et al. 2008, ApJ, 686, 667

Snellen, I. A. G., de Kok, R. J., de Mooij, E. J. W., \& Albrecht, S. 2010, Nature, 465, 1049

Swain, M. R., Vasisht, G., \& Tinetti, G. 2008, Nature, 452, 329

Tinetti, G. 2006, Origins of Life and Evolution of the Biosphere, 36, 541

Tinetti, G., Liang, M.-C., Vidal-Madjar, A., et al. 2007, ApJ, 654, 99

Vidal-Madjar, A., Lecavelier des Etangs, A., Désert, J.-M., et al. 2003, Nature, 422, 143

Vidal-Madjar, A., Désert, J.-M., Lecavelier des Etangs, A., et al. 2004, ApJ, 604, 69

Vidal-Madjar, A., Arnold, L., Ehrenreich, D., et al. 2010, A\&A, 523, A57

Vidal-Madjar, A., Sing, D. K., Lecavelier Des Etangs, A., et al. 2011, A\&A, 527, A110

Wilquet, V., Fedorova, A., Montmessin, F., et al. 2009, J. Geophys. Res. (Planets), 114, E00B42

Yung, Y. L., \& DeMore, W. B. 1999, Photochemistry of Planetary Atmospheres (Oxford University Press) 